\title{
Prognostic significance of negative conversion of high-risk Human Papillomavirus DNA after treatment in Cervical Cancer patients
}

\author{
Qingqing Chen ${ }^{*}$, Runjun Shi ${ }^{*}$, Zhengcao Liu1 ${ }^{*}$, Zhouhong Shi ${ }^{3}, \mathrm{Ke} \mathrm{Gu}^{2}$, Jie Chen ${ }^{1}$, Yan $\mathrm{He}^{1}$, Ying $\mathrm{Li}^{1}$,

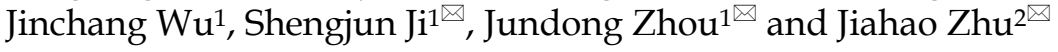

1. Department of Radiotherapy \& Oncology, The Affiliated Suzhou Hospital of Nanjing Medical University, Suzhou, China.

2. Department of Radiotherapy \& Oncology, Affiliated Hospital of Jiangnan University, Wuxi, China.

3. Department of Gynecology, The Affiliated Suzhou Hospital of Nanjing Medical University, Suzhou, China.

*These authors contributed equally to this work.

$\triangle$ Corresponding authors: Shengjun Ji, Department of Radiotherapy \& Oncology, Nanjing Medical University Affiliated Suzhou Hospital, No.16 Baita Road, Suzhou, China, 215001. Tel: +86 18914087033; E-mail: happy168123@aliyun.com; Jundong Zhou, Department of Radiotherapy \& Oncology, Nanjing Medical University Affiliated Suzhou Hospital, No.16 Baita Road, Suzhou, China, 215001. Tel: +86 18962174389; E-mail: szslyyzjd@163.com; Jiahao Zhu, Department of Radiotherapy \& Oncology, Affiliated Hospital of Jiangnan University, 200 Huihe Road, Wuxi, China, 214062. Tel: +86 18851729903; E-mail: zjhmedicine@163.com.

(1) The author(s). This is an open access article distributed under the terms of the Creative Commons Attribution License (https://creativecommons.org/licenses/by/4.0/). See http://ivyspring.com/terms for full terms and conditions.

Received: 2020.04.05; Accepted: 2020.08.01; Published: 2020.08.10

\begin{abstract}
Objective: To evaluate the prognostic value of conversion of high-risk human papillomavirus (HR-HPV) status after treatment for cervical cancer.

Methods: A total of 112 cervical cancer patients with HR-HPV positivity without distant metastasis treated with surgery or radical concurrent radiochemotherapy were enrolled. HR-HPV status was analyzed before and after treatment and at the time point of recurrence or metastasis. Log-rank tests and Cox proportional hazard models were used to evaluate the association between conversion of HR-HPV status after treatment and survival.

Results: Eighty-four (75\%) patients had negative conversion HR-HPV (ncHR-HPV) after treatment and twenty-eight (25\%) were persistent positive HR-HPV (ppHR-HPV). The negative conversion rate was $75.8 \%$ in patients who received surgical treatment and $71.4 \%$ in patients who received radical concurrent radiochemotherapy. There was no significant difference between the two groups $\left(X^{2}=0.000, P=1.000\right)$. There was no significant correlation between HR-HPV conversion after treatment with age $\left(X^{2}=0.616, P=0.252\right)$, FIGO stage $\left(X^{2}=0.051, P=0.823\right)$ and pathological type $\left(X^{2}=0.000, P=1.000\right)$. Univariate analysis showed that treatment regimen and ncHR-HPV was closely related to progression-free survival (PFS) and overall survival (OS) of cervical cancer patients. Multivariate COX regression model showed that treatment regimen $(\mathrm{HR}=3.57,95 \% \mathrm{Cl}: 1.57-8.11, P=0.002)$ and ncHR-HPV $(\mathrm{HR}=5.14,95 \% \mathrm{Cl}: 2.32-11.46, P<0.001)$ were independent prognostic factors for PFS, while only ncHR-HPV $(\mathrm{HR}=12.56,95 \% \mathrm{Cl}$ : 3.54-44.65, $P<0.001)$ was an independent prognostic factor for OS. The presence of PPHR-HPV after treatment $\left(\chi^{2}=14.827, P<0.001\right)$ was associated with recurrence and metastasis. Eleven of the patients with ncHR-HPV after treatment had recurrence or metastasis, and HPV reinfection was not detected in any of them.

Conclusion: ncHR-HPV after treatment in cervical cancer patients indicated better PFS and OS, while PPHR-HPV indicated worse prognosis and high risk of recurrence or metastasis. For patients with ncHR-HPV after treatment, continued HPV screening may not predict recurrence or metastasis. This study suggested that HR-HPV monitoring is necessary for PPHR-HPV patients after treatment but may not be for ncHR-HPV patients. However, further large and multi-center prospective studies should be performed to confirm these findings.
\end{abstract}

Key words: High-risk Human Papillomavirus DNA; Prognosis; Cervical Cancer 


\section{Introduction}

Cervical cancer is one of the most common gynecologic cancers worldwide and causes thousands of cancer-related deaths in women, and there are about 500,000 new cases of cervical cancer in the world each year [1]. China accounts for about onethird of the world's total annual new cases [2]. Despite advances in treatment development during the past decade, $20 \%$ to $40 \%$ of cervical cancer patients will still have local recurrence or distant metastases, especially during the initial two years after treatment [3-4]. As it is well known, the International Federation of Gynecology and Obstetrics stage (FIGO stage) is a prognostic factor of cervical cancer, but significant differences in prognosis are often observed for the same stage. Therefore, it is necessary to seek additional biomarkers with significant clinical prognostic value for cervical cancer.

Infection with human papillomavirus (HPV), especially with high-risk HPV(HR-HPV), including HPV16, 18, 31, 33, 35, 39, 45, 51, 52, 56, 58, 59, 68, 73 and 82 , is the most important risk factor associated with cervical cancer [5-7]. It has been reported that more than $90 \%$ of patients with invasive cervical carcinoma were infected with HPV [8]. In the past years, a large number of studies focused on the correlation between HPV status before treatment and prognosis in patients with cervical cancer [9-13]. Though some studies have shown that cervical cancer patients with HR-HPV positive could become negative after treatment (negative conversion), few studies were concerned with HR-HPV status after treatment. Intharaburan et al. reported that HPV positive after treatment was associated with persistent and recurrent disease and this oncogenic virus may be was a biomarker for pelvic recurrence [14]. However, the prognostic value of the negative conversion HR-HPV (ncHR-HPV) is yet to be reported. Therefore, we assessed the association of the ncHR-HPV with overall survival (OS) and progression free survival (PFS) in cervical cancer patients. Additionally, the association between persistent positive HR-HPV (ppHR-HPV) after treatment and recurrence or metastasis in cervical cancer was evaluated.

\section{Materials \& Methods}

\section{Patients}

For this retrospective study, we collected data of cervical cancer patients who had been diagnosed with HR-HPV positive before treatment in The Affiliated Suzhou Hospital of Nanjing Medical University. Inclusion conditions: (1) Cervical cancer was confirmed by pathology. Patients received surgery or radiotherapy or chemotherapy according to the National Comprehensive Cancer Network (NCCN) Cervical Cancer guidelines. (2) HPV of the patients was positive and the classification was clear before treatment. (3) There is no history of HPV vaccination. (4) No distant metastasis was confirmed by imaging examination. Exclusion criteria: (1) Patients with no pathology. (2) HR-HPV was negative or unclear before treatment. (3) Patients without HPV test after treatment. (4) Patients who lost follow-up and did not follow up regularly. (5) Patients have suffered from other malignant tumors in the past. All patients were confirmed in accordance with the pathologic evidence and had the Karnofsky Performance Status (KPS) of more than 70. Staging was based on the criteria of FIGO 2009. The basic information of these patients was collected, including age, clinical stage, pathological classification, and treatment regimen. Patients were divided into two groups with ncHR-HPV and ppHR-HPV respectively depending on conversion of their HPV status before and after treatment.

All procedures performed were in accordance with the ethical standards of the responsible committee on human experimentation (institutional and national) and with the Helsinki Declaration of 1964 and later versions. This study was approved by the Institutional Review Board of The Affiliated Suzhou Hospital of Nanjing Medical University (No. KL901060). Informed consent was obtained from all individual participants included in the study or their family.

\section{HPV genotyping}

A cervical brush was fully inserted into the cervical canal and the cervical canal was gently rotated five laps clockwise. Cervical exfoliated cells were collected inside and outside the cervix and brush was placed in the preservation solution to prepare for HPV testing. We used HPV nucleic acid amplification test kit for Cape biochemical company (Guangzhou, China). HPV DNA in cervical secretions was detected with hybridization micro array technology (Hybri Max) detection. It Can detect 21 kinds of HPV subtypes, including 15 kinds of high-risk subtype (16, $18,31,33,35,39,45,51,52,56,58,59,68,73$ and 82) and 6 kinds of low-risk subtype $(6,11,42,43,44$, CP8304). All respondents underwent gynecological examination and were collected of cervical secretions by a professional gynecologist. With a cervical brush was fully inserted into the cervical canal, the cervical canal was gently rotated 5 laps by clockwise. Cervical exfoliated cells were collected inside and outside the cervix, and brush was placed in the preservation solution to prepare for HPV testing. Mentioned the use of genome extraction kit DNA, PCR amplification 
(using $\mathrm{AB}$ 17300-based PCR amplification), hybridization, and got HPV typing results obtained by color. HPV in cervical secretions was detected for each patient every three month after treatment and at the time of recurrence or metastasis.

If one or more HPV subtype was positive in 21 kinds of subtypes, it represented HPV DNA testing was positive. All of the negative for HPV DNA was determined to be negative of HPV DNA. If one or more high-risk HPV subtype was positive in 21 kinds of subtypes, it represented high-risk HPV DNA testing was positive.

\section{Treatment}

Patients with early-stage cervical cancer (FIGO stage IB1-IIA) underwent radical hysterectomy and pelvic lymph node dissection. Postoperative pelvic radiotherapy \pm concurrent chemotherapy was administered to patients at high risk for recurrence. Patients with locally advanced cervical cancer (FIGO stage IIB-IV) underwent concurrent radiochemotherapy (CCRT). External beam radiotherapy (EBRT) was delivered to the whole pelvis with 45-50Gy in 25 fractions for the postoperative patients. In the definitive setting, HDR ICR was delivered twice a week in 5 fractions, with a total dose of 30Gy. The prescription of dose was given to point A. The cisplatin with $75 \mathrm{mg} / \mathrm{m}^{2}$ was administered for two courses during radiotherapy.

\section{Follow-up}

Follow-up examinations were performed at regular intervals: three monthly during the first three years, six monthly during the following year. OS was defined as the time between the date of diagnosis and the date of cervical cancer-related death or the last follow-up. PFS was measured from the date of diagnosis to the date of recurrence, distant metastasis, or the last follow-up.

\section{Statistical methods}

The $\chi^{2}$ test was performed to compare categorical variables. Associations between HR-HPV status and OS and PFS were analyzed using Kaplan-Meier curves and were compared using the log-rank test. Multivariate analysis for OS and PFS was performed using Cox proportional hazards regression models. The statistical analyses were performed with SPSS 20.0 statistical software (IBM Corporation, Armonk, NY, USA). A $P$-value $<0.05$ was considered statistically significant.

\section{Results}

\section{Patient characteristics}

In total, 112 patients were included in the current study with their characteristics shown in Table 1. The average age was $53.0 \pm 10.2$ years, with a range of 32 to 88 years. Of these, $69(61.6 \%)$ patients were with early tumor stages (FIGO IA-IIA2), 43 (38.4\%) patients were with advanced tumor stages (FIGO IIB-IV). Furthermore, 91 (56.8\%) patients received surgery and $21(43.2 \%)$ patients received concurrent radiochemotherapy. Some of the patients with advanced tumor stages were still undergoing surgery. A total 25 patients $(22.3 \%)$ had recurrence and metastases, and 9 patients $(8.0 \%)$ died due to disease progression.

\section{Association between ncHR-HPV and clinicopathologic features}

In the total patients, 84 patients had ncHR-HPV (75\%) after treatment. Of the 91 patients who underwent surgery, 69 patients had ncHR-HPV $(75.8 \%)$. The 21 patients who underwent radical concurrent radiochemotherapy, 15 Patients had ncHR-HPV $(71.4 \%)$. There was no significant difference between the two groups $\left(X^{2}=0.000\right.$, $P=1.000)$. There was no significant correlation between HR-HPV conversion after treatment neither with age $\left(X^{2}=0.616, P=0.252\right)$, FIGO stage $\left(X^{2}=0.051\right.$, $P=0.823)$, or pathological type $\left(X^{2}=0.000, P=1.000\right)$ (Table 2).

\section{OS and PFS according to the HR-HPV conversion status}

The Kaplan-Meier method was used to estimate the OS and PFS curves, which were stratified according to the HR-HPV status after treatment (Figures $1 \& 2$ ). The 5-year rate for OS and PFS associated with the HR-HPV conversion status after treatment were calculated. The 5-year OS rate was $94.5 \%$ and $57.1 \%$ in the ncHR-HPV and ppHR-HPV groups, respectively $(\mathrm{HR}=22.821,95 \% \mathrm{CI}=5.142$ to $101.304, P<0.001)$. The 5 -year PFS rate was $82.5 \%$ and $45.2 \%$ in the ncHR-HPV and the ppHR-HPV groups, respectively $(\mathrm{HR}=4.923,95 \% \mathrm{CI}=1.866$ to 12.989 , $P<0.001)$. In brief, the patients with ncHR-HPV had better OS and PFS than those of patients with ppHR-HPV.

\section{Univariate and multivariate cox regression survival analyses}

Univariate analysis showed that treatment regimen $(\mathrm{HR}=0.29,95 \% \mathrm{CI}: 0.10-0.90, P=0.002)$ and ncHR-HPV status (HR=0.21，95\% CI: 0.08-0.53, $P<0.001)$ were closely correlated with PFS in cervical cancer patients. Multivariate COX regression model showed that treatment regimen $(\mathrm{HR}=3.57,95 \% \mathrm{CI}$ : 1.57-8.11, $P=0.002)$ and ncHR-HPV status $(\mathrm{HR}=5.14$, 95\% CI: 2.32-11.46, $P<0.001)$ were independent prognostic factors for PFS (Table 3). 


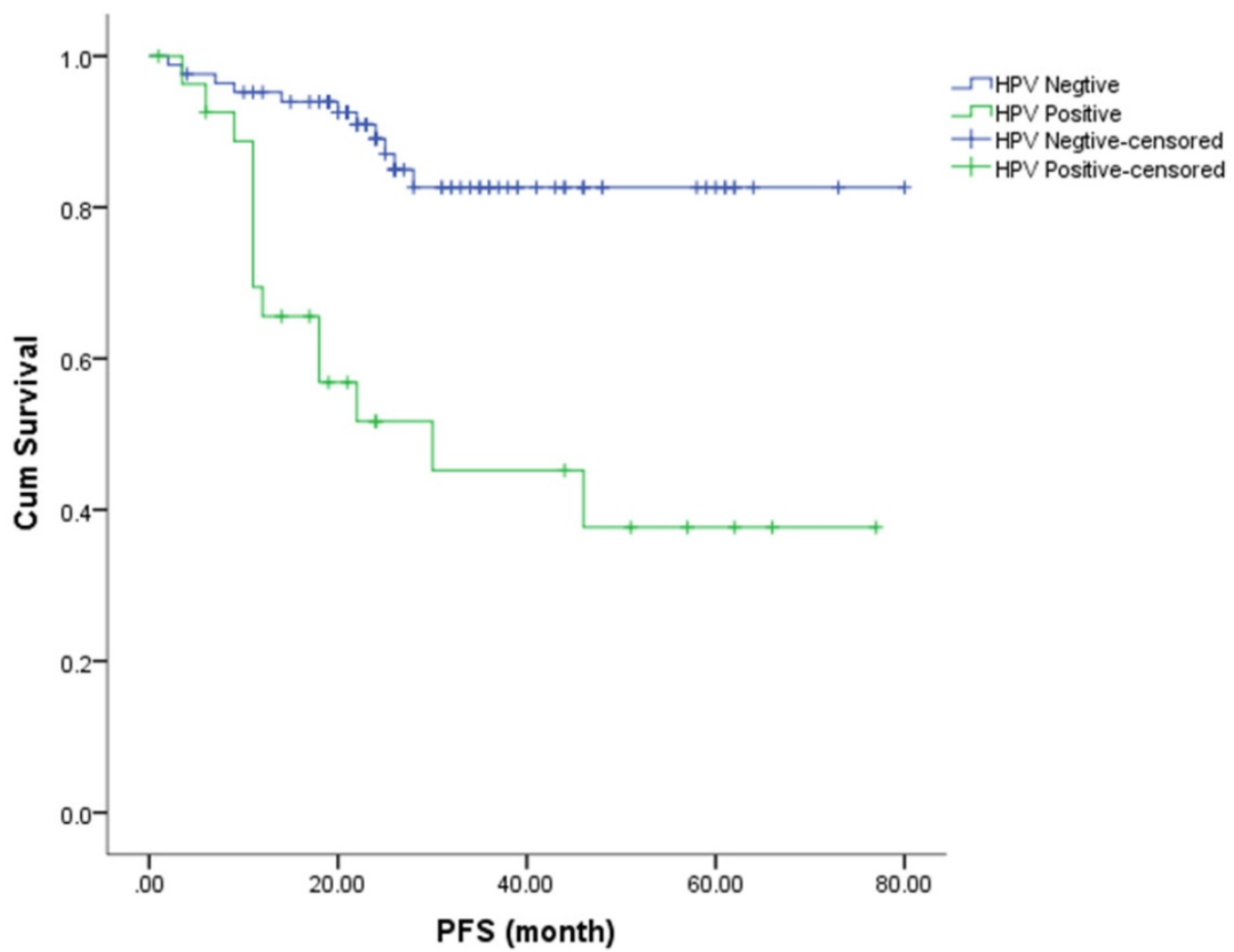

Figure 1. Kaplan-Meier analysis shows the PFS for patients with cervical cancer.

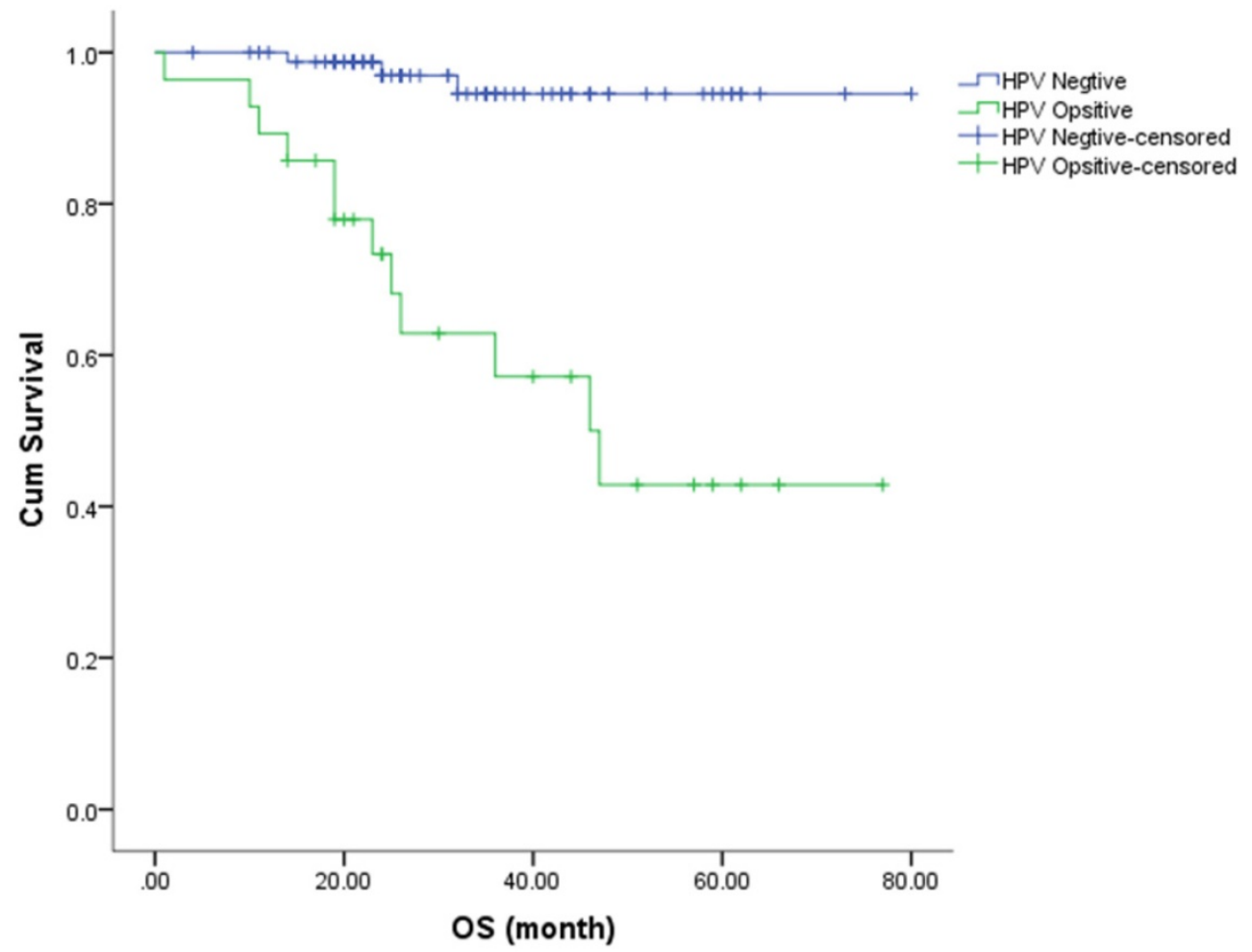

Figure 2. Kaplan-Meier analysis shows the OS for patients with cervical cancer.

Univariate analysis showed that treatment regimen $(\mathrm{HR}=0.35,95 \% \mathrm{CI}$ : $0.10-1.27, P=0.03)$ and ncHR-HPV (HR=0.08, 95\% CI: 0.03-0.26, $P<0.001)$ were closely correlated with OS in cervical cancer patients. Multivariate COX regression model showed that only ncHR-HPV (HR=12.56, 95\% CI: 3.54-44.65, $P<0.001)$ was an independent prognostic factor for OS (Table 4). 
Table 1. Baseline characteristics $(n=112)$

\begin{tabular}{lll}
\hline Variables & $\mathrm{n}$ & $\%$ \\
\hline Age (years) & 30 & \\
$>60$ & 82 & 73.21 \\
$\leq 60$ & & \\
Pathological type & 104 & 92.86 \\
Squamous carcinoma & 5 & 4.46 \\
Adenocarcinoma & 1 & 0.89 \\
Small cell carcinoma & 2 & 1.79 \\
Adenosquamous carcinoma & & \\
FIGO Stage & 69 & 61.61 \\
Stage IA-IIA2 & 43 & 38.39 \\
Stage IIB-IV & & \\
Treatment & 91 & 56.8 \\
Surgery & 21 & 43.2 \\
CCRT & &
\end{tabular}

Table 2. Clinicopathological variables of 112 cervical cancer patients according to HR-HPV conversion status after treatment

\begin{tabular}{lllll}
\hline Variables & ncHR-HPV & ppHR-HPV & $P$ & $\chi^{2}$ \\
\hline Age (years) & & & & \\
$>60$ & $22(19.6)$ & $6(5.4)$ & 0.802 & 0.616 \\
$\leq 60$ & $62(55.4)$ & $22(19.6)$ & & \\
Pathological type & & & & \\
Squamous carcinoma & $78(69.6)$ & $26(23.2)$ & 1.000 & 0.000 \\
Adenocarcinoma & $4(3.6)$ & $1(8.9)$ & & \\
Small cell carcinoma & $0(0)$ & $1(8.9)$ & & \\
Adenosquamous carcinoma & $2(1.8)$ & $0(0)$ & & \\
FIGO Stage (Stage IA-IIA2) & $52(46.4)$ & $18(16.1)$ & 0.823 & 0.051 \\
Stage IIB-IV & $32(26.8)$ & $10(8.9)$ & & \\
Treatment & & & & \\
Surgery & $69(61.6)$ & $23(20.5)$ & 1.000 & 0.000 \\
CCRT & $15(13.4)$ & $5(4.5)$ & & \\
\hline
\end{tabular}

Table 3. Univariate and Multivariate analysis for PFS

\begin{tabular}{|c|c|c|c|c|c|c|}
\hline & \multicolumn{3}{|c|}{ Univariate analysis } & \multicolumn{3}{|c|}{ Multivariate analysis } \\
\hline & HR & $95 \% \mathrm{CI}$ & $P$ & HR & $95 \% \mathrm{CI}$ & $P$ \\
\hline Age $(\leq 60 />60)$ & 0.94 & $0.39-2.28$ & 0.89 & & & \\
\hline $\begin{array}{l}\text { Pathological type } \\
\text { (Squamous/Non- } \\
\text { Squamous carcinoma) }\end{array}$ & 0.85 & $0.18-3.99$ & 0.82 & & & \\
\hline $\begin{array}{l}\text { FIGO Stage } \\
\text { (IA-IIA2/IIB-IV) }\end{array}$ & 0.52 & $0.23-1.18$ & 0.09 & & & \\
\hline $\begin{array}{l}\text { Treatment } \\
\text { (Surgery/CCRT) }\end{array}$ & 0.29 & $0.10-0.90$ & 0.002 & 3.57 & $1.57-8.11$ & 0.002 \\
\hline $\begin{array}{l}\text { HPV status } \\
\text { (nc/ppHR-HPV) }\end{array}$ & 0.21 & $0.08-0.53$ & $<0.001$ & 5.14 & $2.32-11.46$ & $<0.001$ \\
\hline
\end{tabular}

Table 4. Univariate and Multivariate analysis for OS

\begin{tabular}{|c|c|c|c|c|c|c|}
\hline & \multicolumn{3}{|c|}{ Univariate analysis } & \multicolumn{3}{|c|}{ Multivariate analysis } \\
\hline & HR & $95 \%$ CI & $P$ & HR & $95 \% \mathrm{CI}$ & $P$ \\
\hline Age $(\leq 60 />60)$ & 0.53 & $0.18-1.54$ & 0.19 & & & \\
\hline $\begin{array}{l}\text { Pathological type } \\
\text { (Squamous/Non- } \\
\text { Squamous carcinoma) }\end{array}$ & 1.17 & $0.18-7.72$ & 0.88 & & & \\
\hline $\begin{array}{l}\text { FIGO Stage } \\
\text { (IA-IIA2/IIB-IV) }\end{array}$ & 0.46 & $0.17-1.20$ & 0.10 & & & \\
\hline $\begin{array}{l}\text { Treatment } \\
\text { (Surgery/CCRT) }\end{array}$ & 0.35 & $0.10-1.27$ & 0.03 & & & \\
\hline $\begin{array}{l}\text { HPV status } \\
\text { (nc/ppHR-HPV) }\end{array}$ & 0.08 & $0.03-0.26$ & $<0.001$ & 12.56 & $3.54-44.65$ & $<0.001$ \\
\hline
\end{tabular}

Table 5. HR-HPV status after treatment and recurrence or metastasis

\begin{tabular}{llllll}
\hline & $\mathrm{n}$ & $\mathrm{ncHR}-\mathrm{HPV}$ & $\mathrm{ppHR}-\mathrm{HPV}$ & $P$ & $\chi^{2}$ \\
\hline progressive disease & 25 & 12 & 13 & & \\
stable disease & 87 & 74 & 13 & $<0.001$ & 14.827 \\
\hline
\end{tabular}

\section{HR-HPV conversion status and recurrence or metastasis}

Total of 25 patients had recurrence and metastasis, and 13 of them had ppHR-HPV. The presence of ppHR-HPV after treatment $\left(\chi^{2}=14.827\right.$, $P<0.001)$ was associated with recurrence or metastasis (Table 5). Total of 12 patients with ncHR-HPV after treatment had recurrence or metastasis, and in none of them HPV reinfection was found.

\section{Discussion}

In the present study, we evaluated the prognostic value of HR-HPV conversion after treatment for patients with cervical cancer. The results showed that ncHR-HPV after treatment was independent prognostic factor for PFS and OS. The presence of ppHR-HPV after treatment was associated with recurrence and metastasis. Eleven of the patients with ncHR-HPV after treatment had recurrence or metastasis, and HPV reinfection was not detected in any of them. This study suggested that HR-HPV monitoring is necessary for ppHR-HPV patients after treatment but may not be for ncHR-HPV patients.

HPV infection has been identified as a necessary but not sufficient cause of cervical cancer [15]. Persistent and chronic infection with high-risk HPV types (including16, 18, 31, 33, 35, 39, 45, 51, 52, 56, 58, $59,68,73$ and 82) were observed in almost cervical cancers [16]. Over the past decades, a large number of studies focused on the relationship between HPV status and prognosis of cervical cancer [17-20]. Lei J et al [17] performed a nationwide population-based study of cervical cancer cases tested for $13 \mathrm{HR}-\mathrm{HPV}$ types, and found that women with HR-HPV-positive tumors had 39\% lower excess of mortality than women with HR-HPV-negative tumors. Furthermore, a meta-analysis that included 2,838 cases showed that positive HPV DNA before treatment was associated with better OS and PFS than negative HPV DNA in patients with cervical cancer [18]. Besides, Barreto CL [20] reported that the HPV status had no statistically significant effect on the survival of cervical cancer patients. So, there are still controversies about HPV status and prognosis of cervical cancer.

However, few studies focus on the relationship between conversion of HR-HPV status after treatment and prognosis in patients with cervical cancers. Only four studies with good quality assessment involved 
HPV status before and after treatment. However, vast variation in the HPV detection rate was observed in these studies [21]. Methods of HPV detection and radiotherapy may be attributed to the disparities, because three of the four studies were performed 10 years ago. Only one study by Mahantshetty et al. was conducted in the last 3 years, however HPV16/18 was defected but not all the HR-HPV and the follow-up was only 2 years [22]. In our research, we found that ncHR-HPV was associated with better OS and PFS for cervical cancer. The patients with ppHR-HPV demonstrated worse outcome. We speculated that patients with persistent HPV infection may be insensitive to treatment or already have micrometastases. Besides, patients with persistent HPV infection may have increased risk of developing disease recurrence. Nagai et al. reported that persistence of HPV infection was an alarm for disease recurrence after therapeutic conization for CIN 3 [23]. This may be because persistent HPV infection can upregulate the expression of HPV E6/E7 oncogene and thereby initiate carcinogenesis [24]. HPV E6/E7 oncogene can degrade p53 and inactivate $\mathrm{pRb}$, which maintain the growth of tumors.

Since patients whose HR-HPV status was changed to negative had a better prognosis, would that mean that we should need to take interventions for patients with persistent HP-HPV infection? HRHPV persistence has the ability to effectively downmodulate innate immune response and can delay the development of adaptive immunity. Then, delayed immune response to HR-HPV increases the probability to transform epithelial cells. At this stage, elimination of the virus can never prevent cancer development [25-26]. This indicates not only the preventive HPV vaccination, but also therapeutic HPV vaccines, which can induce cellular immune response against HPV oncoproteins and eliminate malignant cells expressing HPV proteins are urgently needed. Currently, several therapeutic vaccines, including recombinant protein vaccines, peptide vaccines, chimeric vaccines, nucleic acid vaccines are being studied for efficacy and safety [27-29]. We look forward to the early clinical use of these vaccines.

In our study, we found that ppHR-HPV after treatment was associated with recurrence or metastasis, which indicated that HR-HPV monitoring is necessary for ppHR-HPV patients after treatment. However, 12 patients with ncHR-HPV after treatment had recurrence and metastasis, but for none of them HPV reinfection was detected at the time of recurrence and metastasis. Therefore, for patients with ncHR-HPV after treatment, continued HPV monitoring may not predict recurrence or metastasis.
More large-scale prospective research should be carried out to explore it.

There were some limitations in this study. Firstly, this study was a retrospective study with a small sample size, so a prospective study with a larger sample size should be needed to further validate the current conclusions. Another limitation of this study was that we did not analyze the impact of different HPV genotype and multiple genotypes of HPV. Chennai et al. reported that infection with multiple genotypes was associated with poor prognosis and early recurrences in comparison to a single genotype [30]. However, this study indicated that molecular detection of HR-HPV conversion status may facilitate early diagnosis of residual and early recurrent cancers after radiotherapy.

\section{Conclusions}

In conclusion, this study demonstrated that ncHR-HPV after treatment in cervical cancer patients indicated better PFS and OS, while ppHR-HPV indicated worse prognosis and high risk of recurrence or metastasis. Therefore, we suggest that HR-HPV monitoring is necessary for ppHR-HPV patients after treatment but may not be for ncHR-HPV patients. However, further large and multi-center prospective studies should be performed to confirm these findings.

\section{Acknowledgements}

This study was supported by Jiangsu Provincial Medical Youth Talent [QNRC2016234]; The Jiangsu 333 Project Scientific Research Funding Program [BRA2018008]; High-level Health Talent "Six One Project" Top Talent Program of Jiangsu Province [LGY2018009]; Suzhou Cancer Clinical Medical Center [Szzx201506].

\section{Ethical Statement}

All procedures performed were in accordance with the ethical standards of the responsible committee on human experimentation (institutional and national) and with the Helsinki Declaration of 1964 and later versions. This study was approved by the Institutional Review Board of The Affiliated Suzhou Hospital of Nanjing Medical University (No. KL901060).

\section{Competing Interests}

The authors have declared that no competing interest exists.

\section{References}

1. Bray F, Ferlay J, Soerjomataram I, et al. Global Cancer Statistics 2018: GLOBOCAN Estimates Of Incidence And Mortality Worldwide for 36 Cancers in 185 Countries. CA Cancer J Clin. 2018; 68(6): 394-424. 
2. Tsu V, Jerónimo J. Saving the World's women from cervical cancer. New Engl J Med, 2016; 374(26): 2509.

3. Kumar L, Harish P, Malik PS, et al. Chemotherapy and targeted therapy in the management of cervical cancer. Curr Probl Cancer. 2018; 42(2): 120-8.

4. Ma L, Liu JM, Zhang J, et al. A pilot study of oral S-1 for treating heavily pretreated patients with advanced or recurrent cervical cancer among Chinese population. Medicine (Baltimore), 2018; 97(22): e10922.

5. Walboomers JM, Jacobs MV, Manos MM, et al. Human papillomavirus is a necessary cause of invasive cervical cancer worldwide. J Pathol. 1999; 189: 12-9.

6. Zur HH. Papillomaviruses and cancer: From basic studies to clinical application. Nat Rev Cancer. 2002; 2(5): 342-50.

7. Bosch FX, Lorincz A, Muñoz N, et al. The causal relation between human papillomavirus and cervical cancer. J Clin Pathol. 2002; 55:244-65.

8. Okuma K, Yamashita H, Yokoyama $\mathrm{T}$, et al. Undetected human papillomavirus DNA and uterine cervical carcinoma: Association with cancer recurrence. Strahlenther Onkol. 2016; 192: 55-62.

9. de Cremoux P, de la Rochefordiere A, Savignoni A, et al. Different outcome of invasive cervical cancer associated with high-risk versus intermediate-risk HPV genotype. Int J Cancer. 2009; 124: 778-82.

10. Rodriguez-Carunchio L, Soveral I, Steenbergen RD, et al. HPV-negative carcinoma of the uterine cervix: a distinct type of cervical cancer with poor prognosis. Bjog. 2015; 122: 119-27.

11. Y Harima, S Sawada, K Nagata, et al. Human Papilloma Virus (HPV) DNA Associated With Prognosis of Cervical Cancer After Radiotherapy. Int J Radiat Oncol Biol Phys. 2002; 52(5): 1345-51.

12. Dabic MM, Nola M, Tomicic I, et al. Adenocarcinoma of the uterine cervix: prognostic significance of clinicopathologic parameters, flow cytometry analysis and HPV infection. Acta Obstet Gynecol Scand. 2008; 87: 366-72.

13. Feng $\mathrm{D}, \mathrm{Xu} \mathrm{H}, \mathrm{Li} \mathrm{X}$, et al. An association analysis between mitochondrial DNA content, G10398A polymorphism, HPV infection, and the prognosis of cervical cancer in the Chinese Han population. Tumour Biol. 2016; 37: 5599-607.

14. Intharaburan $S$, Tanapat $Y$, Jarruwale $P$, et al. Human papillomavirus infection following radiation therapy or concurrent chemoradiation for invasive cervical cancer. J Med Assoc Thai. 2012; 95: S38-S41.

15. de Sanjose S, Quint WG, Alemany L, et al. Human papillomavirus genotype attribution in invasive cervical cancer: a retrospective cross-sectional worldwide study. Lancet Oncol. 2010; 11(11): 1048-56.

16. Crosbie EJ, Einstein $\mathrm{MH}$, Franceschi $\mathrm{S}$, et al. Human papillomavirus and cervical cancer. Lancet 2013; 382: 889-99.

17. Lei J, Ploner A, Lagheden C. High-risk human papillomavirus status and prognosis in invasive cervical cancer: A nationwide cohort study. PLoS Med. $2018 ; 15(10)$ : e1002666.

18. Li P, Tan Y, Tian Y, et al. Prognostic value of HPV DNA status in cervical cancer before treatment: a systematic review and meta-analysis. Oncotarget. 2017; 8(39): 66352-9.

19. Lai $\mathrm{CH}$, Chang $\mathrm{CJ}$, Huang $\mathrm{HJ}$, et al. Role of human papillomavirus genotype in prognosis of early-stage cervical cancer undergoing primary surgery. J Clin Oncol 2007; 25: 3628-34.

20. Barreto CL, Martins DB, de Lima Filho JL, et al. Detection of human papillomavirus in biopsies of patients with cervical cancer, and its association with prognosis. Arch Gynecol Obstet. 2013; 288 (3):643-8.

21. Sabeena S, Kuriakose S, Damodaran B, et al. Human papillomavirus (HPV) DNA detection in uterine cervix cancer after radiation indicating recurrence: a systematic review and meta-analysis. J Gynecol Oncol, 2020; 31: e20.

22. Mahantshetty $U$, Teni $T$, Naga P, et al. Impact of HPV $16 / 18$ infection on clinical outcomes in locally advanced cervical cancers treated with radical radio (chemo) therapy-a prospective observational study. Gynecol Oncol 2018; 148:299-304.

23. Nagai $Y$, Maehama T, Asato T, et al. Persistence of human papillomavirus infection after therapeutic conization for CINIII: Is it an alarm for disease recurrence? Gynecol Oncol. 2000; 79: 294-9.

24. Wang $\mathrm{X}$, Wang $\mathrm{HK}, \mathrm{Li} \mathrm{Y}$, et al. micmRNAs are biomarkers Of oncogenic human papillomavirus infections. Proc Natl Acad Sci U S A. 2014; 111(11): 4262-7.

25. Nunes RAL, Morale MG, Silva GAF, et al. Innate immunity and HPV: friends or foes. Clinics (Sao Paulo). 2018; 73 (Suppl 1), e549s.

26. Stanley M. HPV-immune response to infection and vaccination. Infect Agent Cancer. 2010; 5: 19

27. Ilyinskii PO, Kovalev GI, O'Neil CP, et al. Synthetic vaccine particles for durable cytolytic $\mathrm{T}$ lymphocyte responses and anti-tumor immunotherapy. PLoS One. 2018; 13(6): e0197694.

28. Mukherjee S, Hussaini R, White R, et al. TriCurin, a synergistic formulation of curcumin, resveratrol, and epicatechin gallate, repolarizes tumor-associated macrophages and triggers an immune response to cause suppression of HPV+ tumors. Cancer Immunol Immunother. 2018; 67(5): 761-74.

29. Cordeiro MN, De Lima RCP, Venuti A, et al. Current research into novel therapeutic vaccines against cervical cancer. Expert Rev Anticancer Ther. 2018; 18(4): 365-76.

30. Munagala R, Donà MG, Rai SN, et al. Signifcance of multiple HPV infection in cervical cancer patients and its impact on treatment response. Int J Oncol. 2009; 34:263-71. 